\title{
Optical conductivity \\ associated with solitons in the Peierls state \\ as modified by zero-point-motion disorder
}

\author{
KIHONG KIM* \\ Physics Department, Ajou University, Suwon 442-749, Korea \\ and \\ JOHN W. WILKINS \\ Physics Department, Ohio State \\ University, Columbus, $\mathrm{OH}$ 43210-1168
}

\begin{abstract}
We extend previous work to consider the effect of the soliton on the density of states and conductivity of quasi-one-dimensional Peierls systems with quantum lattice fluctuations, modeled by a random static disorder. Two features have been verified over an order of magnitude variation in the disorder. (1) The soliton density of states and the leading edges of both the soliton-to-band and the band-to-band conductivities have universal scaling forms. (2) The soliton-to-band conductivity has the remarkable feature that the leading edge is accurately predicted by the joint density of states while the trailing edge tracks the rigid-lattice conductivity. Or, in other words, disorder dominates the leading edge, while matrix element effects are predominant for the trailing edge.
\end{abstract}

PACS: 71.20.Hk, 71.45.Lr, 71.55.Jv, 73.20.Dx 


\section{INTRODUCTION}

This paper is the next logical step in working out the consequences of a simple observation for most quasi-one-dimensional systems: the lattice zero-point motion is comparable to the lattice dimerization associated with the Peierls ground state. ${ }^{1}$ The treatment of quantum lattice fluctuations about the dimerized ground state has to date resisted theoretical solution. Below we describe what has been accomplished.

The first step was to model the quantum fluctuations with a static random potential. ${ }^{1}$ This proved possible, for temperatures below the transition temperature, since (i) the characteristic phonon energies are less than the Peierls energy gap parameter $\Delta$ and (ii) the phonons are then effectively dispersionless. At the mathematical level, the gap parameter is supplemented by a complex random variable $\delta \Delta(x)$ with a zero average and

$$
\frac{\left\langle\delta \Delta(x) \delta \Delta^{*}(y)\right\rangle}{\Delta^{2}} \equiv \eta \delta\left((x-y) / \xi_{o}\right) .
$$

By measuring length in terms of the coherence length $\xi_{o} \equiv \hbar v_{F} / \Delta$, we can characterize the static disorder by the dimensionless number $\eta$.

This model can be fitted into the standard continuum form for the electronic part

of the Peierls-Frölich Hamiltonian ${ }^{2,3}$ (see Section III). The density of states $\rho_{\text {disorder }}$ for the resulting problem can be solved analytically, either for the case of an incommensurate lattice distortion ${ }^{4}$ or for the commensurate case of a half-filled band. ${ }^{5}$ Both cases give similar results for weak disorder but differ qualitatively for the experimentally uninteresting case of strong disorder $(\eta>1)$.

In comparison the density of states for the rigid-lattice

$$
\rho_{\text {rigid-lattice }}(E)=\frac{1}{\pi \xi_{o}} \frac{1}{\sqrt{E^{2}-\Delta^{2}}} \frac{|E|}{\Delta} \theta(|E|-\Delta),
$$

with a gap for energies $|E|<\Delta$ has two important features: (1) the square-root singularity 
at $E= \pm \Delta$ is smeared out and (2) the density of states is now finite for all energies and has a substantial subgap tail.

The second step was to extend model (described in detail in Section III) calculations to compute the optical conductivity. For the rigid-lattice the gap in the density of states leads naturally to a gap in the optical conductivity until $\hbar \omega=2 \Delta$ where there is a square-root singularity. In contrast all experiments show a strongly rounded peak about $2 \Delta$, and it was known ${ }^{6}$ that this shape could not be fitted by just rounding the rigid-lattice results.

A complex analytical and numerical calculation ${ }^{7}$ leads to a rounded peak conductivity which resembled existing experimental data. A striking feature was that the conductivity below the absorption peak could be fitted with a universal form ${ }^{8}$ which could be used to deduce the Fermi velocity and $\eta$ from the data. This work has encouraged more data from the experimentalists now able to fit their data. $9,10,11$

The third step - in this paper - is to compute the effect of solitons on the optical conductivity of quasi-one-dimensional compounds. Since solitons are the most studied and discussed feature of these materials, this work is long overdue. As Section III shows a considerable enhancement of the techniques developed before ${ }^{7}$ was required. The next section summarizes the principal conclusions. More extensive results are given in Section IV followed by conclusions.

\section{SUMMARY OF RESULTS}

A. Review of soliton absorption in rigid-lattice system

We use this section both to define notation and to describe the physics of the rigid lattice in a form appropriate for comparing with our results for disordered systems. As 
Heeger et al. ${ }^{12}$ observed a midgap absorption is a universal feature of actual Peierls systems, since solitons can be created by the formation of the material (e.g., in isomerization of cis to trans polyacetylene) or, more usually, by doping the material.

The soliton is located midgap with a wavefunction proportional to $1 / \cosh \left(x / \xi_{o}\right)$ centered in a CDW strand $(-L<x<L)\left[\xi_{o} \equiv \hbar v_{F} / \Delta\right]$. The local density of states is

$$
\begin{aligned}
\rho_{\text {rigid-lattice }}^{\text {soliton }}(E, x) & =\frac{\delta(E)}{\xi_{o}} \frac{1}{2 \cosh ^{2}\left(x / \xi_{o}\right)} \\
& +\frac{1}{\pi \xi_{o}} \frac{1}{\sqrt{E^{2}-\Delta^{2}}}\left(\frac{|E|}{\Delta}-\frac{\Delta}{|E|} \frac{1}{2 \cosh ^{2}\left(x / \xi_{o}\right)}\right) \theta(|E|-\Delta) .
\end{aligned}
$$

The density of states in the absence of the soliton is given by omitting both terms with $\cosh ^{2}\left(x / \xi_{0}\right)$ in $(2.1)$. (Note: $\int_{-\infty}^{\infty} d x \operatorname{sech}^{2}(x) / 2=1$.)

Using this form we could compute the local joint density of states which would indicate an absorption due to the soliton starting at $\Delta$ and localized in the vicinity of the soliton. More accurately one can compute the conductivity from the golden rule. ${ }^{13}$ In particular the matrix element is proportional to the overlap of the continuum state and the soliton wavefunction:

$$
\int \frac{d x}{\xi_{o}} e^{i k x} \operatorname{sech}\left(x / \xi_{o}\right)=\pi \operatorname{sech}\left(\pi k \xi_{o} / 2\right) \equiv \sqrt{2} \psi_{k} .
$$

Using the excitation spectrum above the gap $E(k)=\sqrt{\Delta^{2}+\left(\hbar v_{F}\right)^{2} k^{2}}$ we write

$$
\begin{aligned}
\left.\sigma_{\text {rigid-lattice }}^{\text {soliton }}(\omega)\right|_{\text {soliton-to-band }} & \propto \int d k\left|\psi_{k}\right|^{2} \delta\left(\hbar \omega-E_{k}\right) \\
= & \frac{\xi_{o}}{2 L} \frac{e^{2}}{\hbar} \frac{\xi_{o}}{A} \frac{\pi^{2}}{2} \frac{1}{\sqrt{(\hbar \omega)^{2} / \Delta^{2}-1}} \frac{1}{\cosh ^{2}\left(\pi \sqrt{(\hbar \omega)^{2} / \Delta^{2}-1} / 2\right)}
\end{aligned}
$$

In the last line we give a dimensionally correct form by including the "cross-sectional area" $A$ of the one-dimensional strand. For reference we note that the integrated weight of the soliton conductivity is $2.83\left(e^{2} / \hbar\right)\left(\xi_{o}^{2} /(2 L A)\right)$. Most importantly, the conductivity is proportional to the density of solitons $\xi_{o} /(2 L)$.

The dashed line in Figure 1 shows the soliton-to-band conductivity. We note for reference there is no analytic form for the band-to-band conductivity. Following many 
workers $^{14}$ we use an approximate form for this conductivity

$$
\left.\sigma(\omega)_{\text {rigid-lattice }}^{\text {soliton }}\right|_{\text {band-to-band }}=\frac{e^{2}}{\hbar} \frac{\xi_{o}}{A}\left(\frac{2 \Delta}{\hbar \omega}\right)^{2} \frac{2 \Delta}{\sqrt{(\hbar \omega)^{2}-(2 \Delta)^{2}}}\left(1-\frac{\xi_{o}}{L}\right) .
$$

Equations (2.4) and (2.5) will be used for comparion with our results.

\section{B. Sample result for absorption in disordered system}

As discussed in the introduction, the effect of the zero-point motion is approximated by a static disorder which is characterized by a dimensionless parameter $\eta$. In section III we will give the details of the procedure by which we compute the disordered case. The important point is that the calculation gives results which could not be anticipated by just smearing the rigid-lattice case - that is, with no disorder. As noted in the introduction, in the calculation of CDW conductivity, ${ }^{7}$ such a convolution gives a large low frequency conductivity that is not observed and does not even reproduce the qualitative shape of the frequency-dependent conductivity seen experimentally.

Figure 2a shows the soliton density of states for rigid-lattice and the disordered-lattice with a range of $\eta$ 's. In Section IV we will discuss curves for a variety of $\eta$ and show how the soliton peak can be scaled to deduce the dependence of the width on $\eta$. Here we concentrate on the main features. The most important feature is the delta function midgap state (2.1) is broadened. This broadening has two consequences: (1) the soliton-to-band absorption will certainly be broadened on the leading edge and (2) the conductivity will be affected by the doping or Fermi level. When the Fermi level is tied to the middle of the soliton peak, only filled soliton states below the Fermi level can have transitions to the empty conduction states. On the other hand, if the Fermi level lies wholly above the soliton band, then the whole soliton band can have transitions to the conduction band, thus leading to a broader absorption than when the Fermi level is pinned to the midgap. This effect is real and should be observable. It is discussed in detail in Section IV.B. 
In Figure 1 the solid line shows the conductivity for the disordered lattice with the same value of $\eta=0.01$ as for the narrowest density of states in Fig. 2. Comparing with the dashed curve for the rigid-lattice we see that the leading edge is broadened as expected. In contrast the trailing edge is not appreciably broadened relative to the rigid-lattice case. We will return to this in the next subsection. In section IV there are more detailed comparisons.

\section{Comparison of rigid-lattice and disordered case}

We consider one more curve in Figure 1, the dotted line for the the joint density of states

$$
\rho_{J D O S}^{\text {soliton }}(\omega)=\int d E \rho_{\text {disorder }}^{\text {soliton }}(E) \rho_{\text {disorder }}^{\text {soliton }}(E+\hbar \omega)
$$

Figure 3 shows the limits of the integration for transitions involving the soliton are such to permit two sets of transitions: (a) from the filled part of soliton band to empty conduction band and (b) from filled valence band to the empty part of soliton band. In the case where the Fermi level lies in the soliton band both transitions are possible and lead to a broader joint density of states. In the case where the Fermi level is wholly above (below) the soliton band only case a (b) is permitted and leads to a narrower joint density of states.

The dotted curve in Figure 1 is the joint density of states for $\eta=0.01$ with the Fermi level pinned to the midgap. The striking feature - nearly invisible - is that the leading edges of the joint density of states are nearly coincident with that of the full calculation. This is a common feature for all $\eta$ and all chemical potentials (c.f. Section IV). This suggests that the matrix element is essentially constant for the leading edge. On the other hand, for the trailing edge the dotted line lies clearly above the full calculations. Indeed 
as noted in the previous section the trailing edge tracks the rigid-lattice case which is dominated by the matrix element. In summary,

1. The leading edge is dominated by the joint density of states for the disordered lattice.

2. The trailing edge is dominated by full conductivity for the rigid lattice case.

We have no simple explanation for relatively sudden switch on at $\hbar \omega=\Delta$ of the matrix element effects and relatively sudden switch off of the disorder effects. But we can affirm that it has been seen in extensive numerical calculations over the parameter ranges $0.01<$ $\eta<0.5$ and $0<E_{F}<0.5 \Delta$.

\section{METHOD}

We consider the standard continuum model of noninteracting electrons in one dimension with a Peierls ground state. Our system is described by a Dirac-type equation for the wave functions $\psi_{1}(x)$ and $\psi_{2}(x)$ for electrons moving with the Fermi velocity $v_{F}$ to the right and to the left, respectively, in the interval $-L \leq x \leq L$ with a complex random potential $\delta \Delta(x)$ :

$$
\begin{gathered}
\left(\begin{array}{cc}
-i \hbar v_{F} \frac{\partial}{\partial x} & \Delta(x)+\delta \Delta(x) \\
\Delta(x)+\delta \Delta^{*}(x) & i \hbar v_{F} \frac{\partial}{\partial x}
\end{array}\right)\left(\begin{array}{l}
\psi_{1}(x) \\
\psi_{2}(x)
\end{array}\right)=E\left(\begin{array}{l}
\psi_{1}(x) \\
\psi_{2}(x)
\end{array}\right) \\
\langle\delta \Delta(x)\rangle=\langle\delta \Delta(x) \delta \Delta(y)\rangle=0,\left\langle\delta \Delta(x) \delta \Delta^{*}(y)\right\rangle=\eta \Delta^{2} \delta\left((x-y) / \xi_{o}\right) .
\end{gathered}
$$

In terms of the self-consistent disorder-averaged Peierls gap $\Delta$ in the ground state and the coherence length $\xi_{o}=\hbar v_{F} / \Delta$, the soliton configuration for the spatially-varying gap function $\Delta(x)$ is

$$
\frac{\Delta(x)}{\Delta}=\tanh \left(\frac{x}{\xi_{o}}\right)
$$


We use $G^{+}\left(G^{-}\right)$, the retarded (advanced) $2 \times 2$ matrix Green function, to compute all properties of interest. The density of states is

$$
\rho(E)=\frac{1}{2 L} \int_{-L}^{L} d x\left\langle-\frac{1}{\pi} \operatorname{Im}\left[\operatorname{Tr} G^{+}(x, x \mid E)\right]\right\rangle .
$$

The real part of the frequency-dependent conductivity for a strand of cross-sectional area $A$ at low temperatures $\left(\hbar \omega \gg k_{B} T\right)$ is

$$
\begin{aligned}
\sigma(\omega)= & \frac{e^{2}}{A \hbar} \frac{2}{\pi \hbar \omega} \int_{-\hbar \omega+E_{F}}^{E_{F}} d E \frac{1}{2 L} \int_{-L}^{L} d y \\
& \int_{y}^{\infty} d x \operatorname{Re}\left[j^{+-}(E+\hbar \omega, E \mid x, y)-j^{++}(E+\hbar \omega, E \mid x, y)\right] .
\end{aligned}
$$

The correlation function in (3.5) involves a trace of Green functions and the Pauli matrix $\sigma_{3}$

$$
j^{ \pm \pm}\left(E^{\prime}, E \mid x, y\right)=\left(\hbar v_{F}\right)^{2}\left\langle\operatorname{Tr}\left[\sigma_{3} G^{ \pm}\left(y, x \mid E^{\prime}\right) \sigma_{3} G^{ \pm}(x, y \mid E)\right]\right\rangle .
$$

Finally we compute the conductivity using the approximation $\langle G G\rangle \approx\langle G\rangle\langle G\rangle$.

Two linearly independent wave functions $\psi$ and $\tilde{\psi}$ satisfy the boundary conditions that $\psi(-L)$ and $\tilde{\psi}(L)$ do not diverge as $L$ becomes large, whereas $\psi(L)$ and $\tilde{\psi}(-L)$ diverge. These $\psi$ and $\tilde{\psi}$ will be statistically independent in the limit $L \rightarrow \infty .{ }^{15}$ For $x>y$,

$$
G(x, y \mid E)=\frac{i}{\hbar v_{F}\left(\psi_{1} \tilde{\psi}_{2}-\psi_{2} \tilde{\psi}_{1}\right)}\left(\begin{array}{cc}
\tilde{\psi}_{1}(x) \psi_{2}(y) & \tilde{\psi}_{1}(x) \psi_{1}(y) \\
\tilde{\psi}_{2}(x) \psi_{2}(y) & \tilde{\psi}_{2}(x) \psi_{1}(y)
\end{array}\right),
$$

where $\left(\psi_{1} \tilde{\psi}_{2}-\psi_{2} \tilde{\psi}_{1}\right)$ is a constant independent of $x$. We obtain $G^{+}\left(G^{-}\right)$by solving for wave functions with $\operatorname{Im} E$ a small positive (negative) number. Then the denominator can be Taylor-expanded and

$$
G^{+}(x, y \mid E)=-\frac{i}{\hbar v_{F}} \sum_{n=0}^{\infty}\left(\begin{array}{cc}
\hat{C}_{n}(x, y) \hat{z}_{n}(x) & \hat{D}_{n}(x, y) \hat{z}_{n}(x) \\
\hat{C}_{n}(x, y) \hat{z}_{n+1}(x) & \hat{D}_{n}(x, y) \hat{z}_{n+1}(x)
\end{array}\right),
$$

where

$$
\begin{aligned}
& \hat{C}_{n}(x, y)=\hat{y}_{n}(x) \frac{\psi_{2}(y)}{\psi_{2}(x)}, \hat{D}_{n}(x, y)=\hat{y}_{n}(x) \frac{\psi_{1}(y)}{\psi_{2}(x)}, \\
& \hat{y}_{n}(x)=\left[\frac{\psi_{1}(x)}{\psi_{2}(x)}\right]^{n}, \hat{z}_{n}(x)=\left[\frac{\tilde{\psi}_{2}(x)}{\tilde{\psi}_{1}(x)}\right]^{n} .
\end{aligned}
$$


Next, we take the $L \rightarrow \infty$ limit and utilize the statistical independence of $\psi$ and $\tilde{\psi}$ to factor the average of the Green function into products of averages of $\hat{C}(\hat{D})$ and $\hat{z}$. In general, the quantities $C_{n}(x, y) \equiv\left\langle\hat{C}_{n}(x, y)\right\rangle$ and $D_{n}(x, y) \equiv\left\langle\hat{D}_{n}(x, y)\right\rangle$ are functions of $x$ and $y$, and $y_{n}(x) \equiv\left\langle\hat{y}_{n}(x)\right\rangle$ and $z_{n}(x) \equiv\left\langle\hat{z}_{n}(x)\right\rangle$ are functions of $x$. Thus the averaged Eq. (3.8) can be written in terms of $z_{n}(x), C_{n}(x, y)$ and $D_{n}(x, y)$. The following equations for $y_{n}, z_{n}$ and $C_{n}$ can be derived from Eq. (3.1) using standard Fokker-Planck methods, ${ }^{16}$ subject to the conditions $y_{0}=z_{0}=1, d y_{n} /\left.d x\right|_{x= \pm \infty}=d z_{n} /\left.d x\right|_{x= \pm \infty}=0$, and $C_{n}(x, x)=y_{n}(x)$, which follow from Eq. (3.9) and the fact that the translational invariance has to be restored far from $x=0$ where the soliton is located:

$$
\begin{aligned}
\xi_{o} \frac{d y_{n}}{d x}= & 2 i n \frac{E}{\Delta} y_{n}-i n \frac{\Delta(x)}{\Delta} y_{n+1}-i n \frac{\Delta(x)}{\Delta} y_{n-1}-\eta n^{2} y_{n}, \\
-\xi_{o} \frac{d z_{n}}{d x}= & 2 i n \frac{E}{\Delta} z_{n}-i n \frac{\Delta(x)}{\Delta} z_{n+1}-i n \frac{\Delta(x)}{\Delta} z_{n-1}-\eta n^{2} z_{n}, \\
\xi_{o} \frac{d C_{n}(x, y)}{d x}= & i(2 n+1) \frac{E}{\Delta} C_{n}-i(n+1) \frac{\Delta(x)}{\Delta} C_{n+1}-i n \frac{\Delta(x)}{\Delta} C_{n-1} \\
& -\eta\left(n^{2}+n+\frac{1}{2}\right) C_{n} .
\end{aligned}
$$

The function $D_{n}$ satisfies the same equation as $C_{n}$ with the initial condition $D_{n}(x, x)=$ $y_{n+1}(x)$.

We can relate $y_{n}$ and $z_{n}$ in a simple manner. In the soliton case with $\Delta(x)=-\Delta(-x)$, we can easily prove that $z_{n}(x)=(-1)^{n} y_{n}(-x)$. In order to integrate Eq. (3.10) numerically, we choose a starting point $(x=-L)$ sufficiently far from $x=0$ that the spatial variation of $y_{n}$ is negligible. Then $y_{n}$ satisfy a set of algebraic equations:

$$
\left(2 \frac{E}{\Delta}+i \eta n\right) y_{n}-y_{n+1}-y_{n-1}=0
$$

which we solve by truncating the number of equations. With the solutions of Eq. (3.13) as initial conditions, we integrate Eq. (3.10) from $x=-L$ to $+L$. These results are used as initial conditions for Eq. (3.12). After we obtain $y_{n}(x), z_{n}(x), C_{n}(x, y)$ and $D_{n}(x, y)$, 
and therefore $G^{+}(x, y \mid E)$ for $x>y$ and $E>0$, we get all other Green functions using the symmetry relations

$$
\begin{aligned}
& G_{11}^{ \pm}(y, x \mid E)=G_{22}^{ \pm}(x, y \mid E)=\left[G_{11}^{\mp}(x, y \mid E)\right]^{*}, \\
& G_{12}^{ \pm}(y, x \mid E)=G_{12}^{ \pm}(x, y \mid E)=\left[G_{21}^{\mp}(x, y \mid E)\right]^{*}, \\
& G_{11}^{ \pm}(x, y \mid-E)=-G_{22}^{\mp}(x, y \mid E), \\
& G_{12}^{ \pm}(x, y \mid-E)=G_{21}^{\mp}(x, y \mid E) .
\end{aligned}
$$

The key to our method is the fact that in disordered systems, $y_{n}, z_{n}, C_{n}$, and $D_{n}$ all decay very rapidly as a function of $n$. To solve Eqs. (3.10-13) numerically, we truncate the number of equations by setting $y_{n}=z_{n}=0$ for $n>N$. The results presented here was obtained for $N=40$, which was sufficiently large to ensure convergence. Our system size is 20 coherence lengths, and therefore the soliton density is $1 /\left(20 \xi_{o}\right)$. In the numerical threedimensional integration of Eq. (3.5), we used the mesh sizes of $d E=0.025 \Delta, d x=0.05 \xi_{o}$, and $d y=0.25 \xi_{o}$. Convergence of the data with respect to these parameters was also carefully checked.

\section{RESULTS}

While the basic result was presented in Section II, here we present additional details on pertinent features. Among these are role of (a) disorder on the density of states, (b) chemical potential on the conductivity and (c) disorder on the soliton-induced conductivity.

A. Disorder effect on density of states: scaling

Figure $2 \mathrm{a}$ shows the density of states for values of the disorder from $0.01<\eta<0.08$. The dotted line gives the density of states in the absence of disorder (Eq. (2.1)); of course 
the delta function at $E=0$ cannot be exhibited. We see that the curves evolve smoothly with increasing $\eta$.

Figure $2 \mathrm{~b}$ shows the scaling of the soliton band: $\rho(E, \eta) / \rho(E=0, \eta)$ versus $E / \Gamma(\eta)$ where the half-width $\Gamma(\eta)$ has to be deduced by fitting. Except for the largest $\eta(0.15$ and 0.2) all curves lie on a universal shape which is nearly Gaussian, as evidenced by the fact that $\rho(E=0, \eta) \Gamma(\eta)$ varies less than one-half percent over the full $\eta$ range. The insert in Figure $2 \mathrm{~b}$ shows the variation of $\Gamma(\eta)$ with $\eta$. The dotted line shows the efforts to do a similar scaling for the leading edge of the band gap. Within the scaling the two widths are very similar.

\section{B. Chemical potential: effect on conductivity}

Figure 4 shows how the chemical potential can broaden and shift the conductivity and, in the process, shows that the leading edge is predicted by the joint density of states while the trailing edge reflects the conductivity without disorder. There are two sets of three curves: for the chemical potential $=0$ and $=0.55 \Delta$. Each set has a solid line for the full conductivity, the dotted line for the joint density of states and dashed curve for the rigid-lattice conductivity. Figure 3 shows that the effect of moving the chemical potential from the middle of the soliton band to wholly above it would broaden the soliton-to-band conductivity. Measuring at half height the width from $\hbar \omega=\Delta$, we see the width is roughly doubled as the chemical potential moves from midgap to fully above the soliton band. As striking is how well the joint density of states mimics the calculated conductivity. This further demonstrates that the leading edge of the conductivity bears no effect of any energy dependent matrix element. Less impressive is the agreement of the trailing edge with the conductivity for the soliton without disorder (dashed line).

Only space constraints prevent us from showing similar curves for a wide range of 
$\eta$. Of course the effect is less dramatic as the leading edge broadens but the agreement between $\rho_{J D O S}$ with the leading edge of the conductivity is common feature over the range $0.01<\eta<0.1$.

\section{Disorder effect on conductivity}

Figure 5 illustrates the conductivity for four $\eta$ values $(0.01,0.02,0.05$ and 0.1$)$. While the leading edges of the soliton-to-band and the band-to-band broaden as expected, the trailing edges of both are essentially unchanged, tracking the conductivity of the rigidlattice result, shown by the dotted line. While the curve is for the chemical potential $E_{F}=$ $0.55 \Delta$, the results for the midgap chemical potential are similar. Figure $5 \mathrm{~b}$ demonstrates that the leading edges of the soliton-to-band conductivities have a universal shape when rescaled. The insert shows the $\eta$ dependence of the conductivity half-width $\Gamma_{c}(\eta)$. A very similar scaling exists for the width of the leading edge of the band-to-band conductivity (dotted line in insert).

\section{DISCUSSION AND CONCLUSIONS}

First we should point out all the failings of the calculation. These are as follows: (1) We approximate $\langle G G\rangle=\langle G\rangle\langle G\rangle$. While in principle we know how to overcome this, the set of differential equations to be solved is sufficiently complex that we have not undertaken it. (2) We have used the rigid-lattice results for the variation in $\Delta(x)$ for the soliton. Once we have introduced disorder we should self-consistently solve the soliton energy and wavefunction. This admirable task is also numerically possible, but we have not attempted it. (3) We approximate the quantum fluctuation of the lattice motion by a random static disorder. We believe this is a good approximation in the low-temperature regime but have 
no hard estimate of the error. (4) We have ignored any electron-electron contribution to any of the density of states or conductivities. We would dearly love to consider a tractable model that includes them.

There is another problem with our startling result that there is no energy-dependent matrix-element effect on the leading edge of the soliton-to-band conductivity. It is solely the results of numerical calculation. We cannot yet produce an analytic or physical argument. Given that we have been working of the ramifications of the large-zero point motion since late 1991, we do not see a way to this clearly desirable deeper understanding. We welcome the advice and comments of colleagues in our continuing efforts.

Nonetheless we think there is a very interesting result - the turn off of the disorder effects and and the turn on of the matrix-element effects at $\hbar \omega \sim \Delta-$ which is aptly summarized at the end of Section II. We would strongly encourage experimentalists to produce better data on soliton-to-band conductivity on better samples. We have been impressed by the high-quality data ${ }^{9,10,11}$ our earlier work ${ }^{7}$ on the optical conductivity of the Peierls state induced.

Finally we suggest one way that offers an approximate approach to a parameter-free analysis. In our earlier calculation ${ }^{7}$ for the conductivity of the Peierls state, it was possible to extract $\eta$ from the scaled width of the leading edge of the conductivity. In this paper we have extended the calculations to smaller $\eta$ 's $(0.01-0.1)$ than in our earlier work $(0.1-$ 1.0). The widths for the leading edge of the band-to-band conductivity with and without a soliton roughly agree. Accordingly the measured band-to-band width $\left(\Gamma(\eta) / \Delta=0.83 \eta^{0.60}\right)$ could be used to extract an $\eta$ that in turn could predict the soliton-to-band conductivity.

This work was supported by the KOSEF (Grant No. 951-0209-030-1), Ajou University Research Fund, and the DOE - Basic Energy Sciences, Division of Materials Sciences. Cray time on the Ohio Supercomputer Center is deeply appreciated. 


\section{REFERENCES}

*Electronic mail address: khkim@madang.ajou.ac.kr

1. R. H. McKenzie and J. W. Wilkins, Phys. Rev. Lett. 69, 1085 (1992); Synth. Met. 55-57, 4296 (1993).

2. S. A. Brazovskii and I. E. Dzyaloshinskii, Zh. Eksp. Theor. Fiz. 71, 2338 (1976) [Sov. Phys. JETP 44, 1233 (1976)].

3. H. Takayama, Y. R. Lin-Liu and K. Maki, Phys. Rev. B 21, 2388 (1980).

4. L. P. Gor'kov and O. I. Dorokhov, Fiz. Nizk. Temp. 4, 332 (1978) [Sov. J. Low Temp. Phys. 4, 160 (1978)]; A. Abrikosov and E. A. Dorotheyev, J. Low Temp. Phys. 46, 53 (1982).

5. A. A. Ovchinnikov and N. S. Érikhman, Zh. Eksp. Theor. Fiz. 73, 650 (1977) [Sov. Phys. JETP 46, 340 (1977)]; H. J. Fischbeck and R. Hayn, Phys. Status Solidi (b) 158, 565 (1990).

6. Z.-B. Su and L. Yu, Comm. Theor. Phys. (Beijing) 2, 1341 (1983).

7. K. Kim, R. H. McKenzie and J. W. Wilkins, Phys. Rev. Lett. 71, 4015 (1993).

8. Below the absorption peak $\sigma\left(\omega_{\text {peak }}\right)$, the conductivity can be fitted with the function $\sigma(\omega) / \sigma\left(\omega_{\text {peak }}\right)=\exp \left[-0.49\left|\left(\omega-\omega_{\text {peak }}\right) / \Gamma(\eta)\right|^{2}-0.20\left|\left(\omega-\omega_{\text {peak }}\right) / \Gamma(\eta)\right|^{3}\right]$ and $\Gamma(\eta) / \omega_{\text {peak }}=\eta^{0.62}(0.414+0.077 \eta)$.

9. L. Degiorgi, G. Grüner, K. Kim, R. H. McKenzie and P. Wachter, Phys. Rev. B 49, 14754 (1994).

10. L. Degiorgi, St. Thieme, B. Alavi, G. Grüner, R. H. McKenzie, K. Kim and F. Levy, Phys. Rev. B 52, 5603 (1995). 
11. K. A. Coplin, S. Jasty, S. M. Long, S. K. Manohar, Y. Sun, A. G. MacDiarmid and A. J. Epstein, Phys. Rev. Lett. 72, 3206 (1994) [pernigraniline]; N. Kuroda, M. Nishida and M. Yamashita, private communication [PtCl].

12. A. J. Heeger, S. Kivelson, J. R. Schrieffer and W.-P. Su, Rev. Mod. Phys. 60, 781 (1988).

13. K. Fesser, A. R. Bishop and D. K. Cambell, Phys. Rev. B 27, 4804 (1983).

14. N. Suzuki, M. Ozaki, S. Etemad, A. J. Heeger and A. G. MacDiarmid, Phys. Rev. Lett. 45, 1209 (1980); K. Maki and M. Nakahara, Phys. Rev. B 23, 5005 (1981); J. T. Gammel and J. A. Krumhansl, Phys. Rev. B 24, 1036 (1981); S. Kivelson, T. K. Lee, Y. R. Lin-Liu, J. Peschel and L. Yu, Phys. Rev. B 25, 4172 (1982); J. C. Hicks and A. L. Wasserman, Phys. Rev. B 29, 808 (1984).

15. A. A. Ovchinnikov and N. S. Érikhman, Zh. Eksp. Theor. Fiz. 78, 1448 (1980) [Sov. Phys. JETP 51, 728 (1980)].

16. I. M. Lifshits, S. A. Gredeskul and L. A. Pastur, Introduction to the Theory of Disordered Systems (Wiley, New York, 1988), p. 146. 


\section{FIGURE CAPTIONS}

Fig. 1. Frequency-dependent conductivity for system of one soliton in a Peierls state for a one-dimensional chain of length $20 \xi_{o}$. Solid line corresponds to disorder parameter $\eta=0.01$, while dashed line to rigid-lattice $(\eta=0)$. Dotted curve is the joint density of states for $\eta=0.01$, scaled to match conductivity peak at $E=\Delta$. Note leading edge of conductivity is indistinguishable from joint density of states while trailing edge is similar to rigid-lattice case. These two features are seen for all disorder parameters and for all chemical potentials (doping levels). In this case, chemical potential lies in the center of soliton band. Small structure at $\hbar \omega=0$ is a numerical artifact.

Fig. 2. Density of states for system of one soliton centered in $20 \xi_{o}$ chain of Peierls state for disorder parameters $\eta=0$ (dotted line), 0.01, 0.02, 0.04 and 0.08 . The widths of the soliton and band density of states increase with $\eta$. In (b) the scaled soliton density of states $\rho(E, \eta) / \rho(E=0, \eta)$ is plotted versus $E / \Gamma(\eta)$, thus demonstrating the universal shape for $\eta<0.1$. Only large disorder $(\eta=0.15,0.2)$ cases break away from universal shape for $E / \Gamma(\eta)>2$. The insert shows the $\eta$ dependence of $\Gamma(\eta) / \Delta$ for both soliton band (solid line: $0.465 \eta^{0.49}$ ) and leading band edge (dotted line).

Fig. 3. Schematic plots of the transitions involved in the joint density of states for the $E_{F}=0$ and $0.55 \Delta$. The symbols s-b and b-b correspond to soliton-to-band and band-toband transitions. Since the $E_{F}=0$ soliton-to-band transition covers only half the soliton band, that joint density of states will be roughly half as wide as the $E_{F}=0.55 \Delta$ curve as reflected in Fig. 3 .

Fig. 4. Fermi-energy dependence of the soliton-to-band conductivity for disorder parameter $\eta=0.01$. For each solid line, corresponding to $E_{F}=0$ and $0.55 \Delta$, there is a nearly coincident joint density of states for the same chemical potential. This coincidence is seen for the full range of $\eta$ values. The rigid-lattice conductivity (dashed line) does not track 
the trailing edge of the disordered-lattice conductivity with the same fidelity as the joint density of states does the leading edge.

Fig. 5. Conductivity for disorder parameters $\eta=0$ (dotted line), 0.01, 0.02, 0.05 and 0.1; all for $E_{F}=0.55 \Delta$. In (a) note that rigid-lattice case (dotted) closely tracks the trailing edges of all the disordered-lattice cases. In (b) the scaled soliton-to-band conductivity $\sigma(\omega) / \sigma_{\text {peak }}(\eta)$ is plotted versus $\left(\omega-\omega_{\text {peak }}\right) / \Gamma_{c}(\eta)$, thus demonstrating the universal shape for the leading edge. The insert shows the $\eta$ dependence of $\Gamma_{c}(\eta) / \Delta$ for both soliton-toband (solid line: $0.685 \eta^{0.513}$ ) and band-to-band (dotted line). 


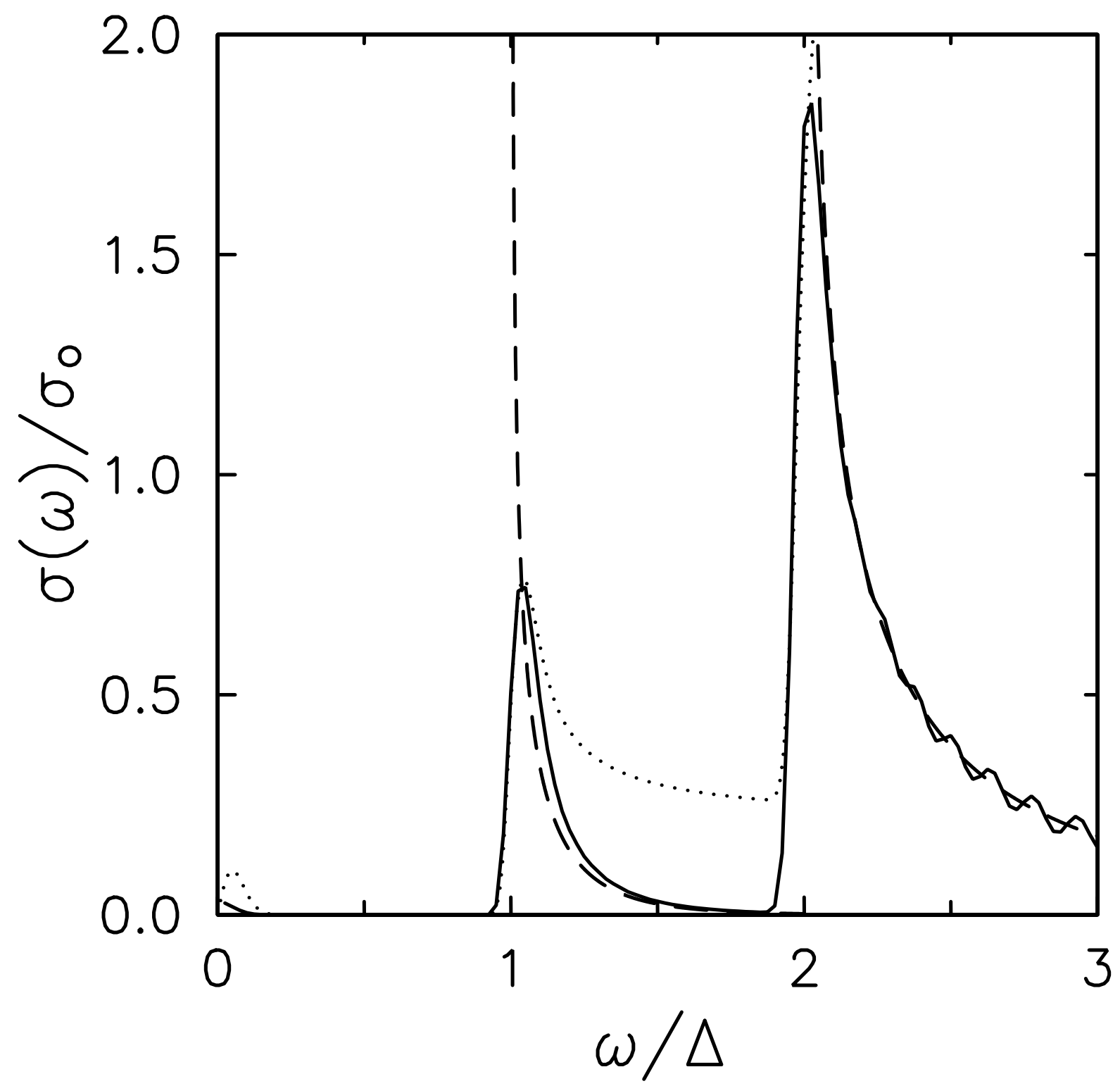

Fig. 1 

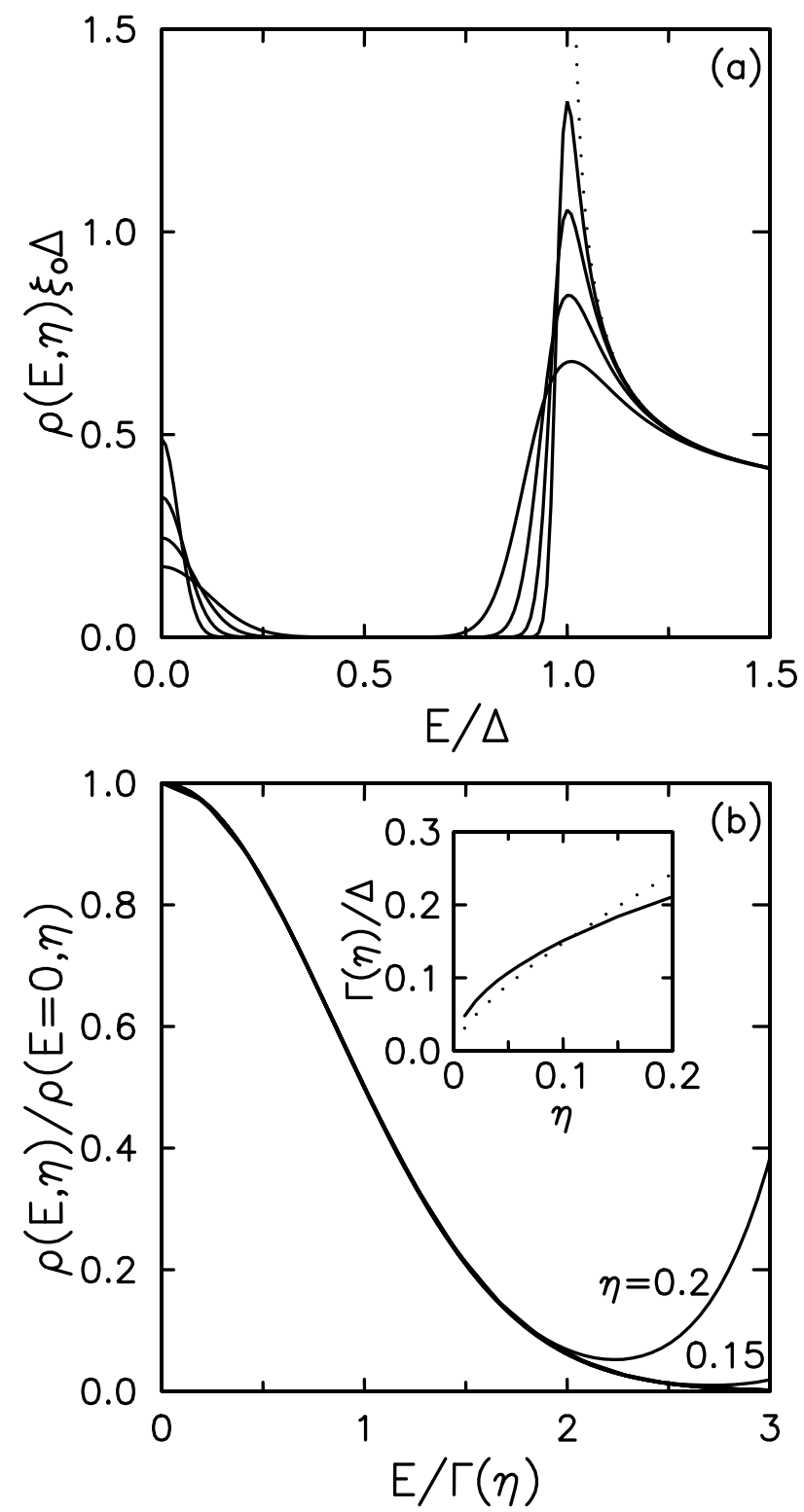

Fig. 2 

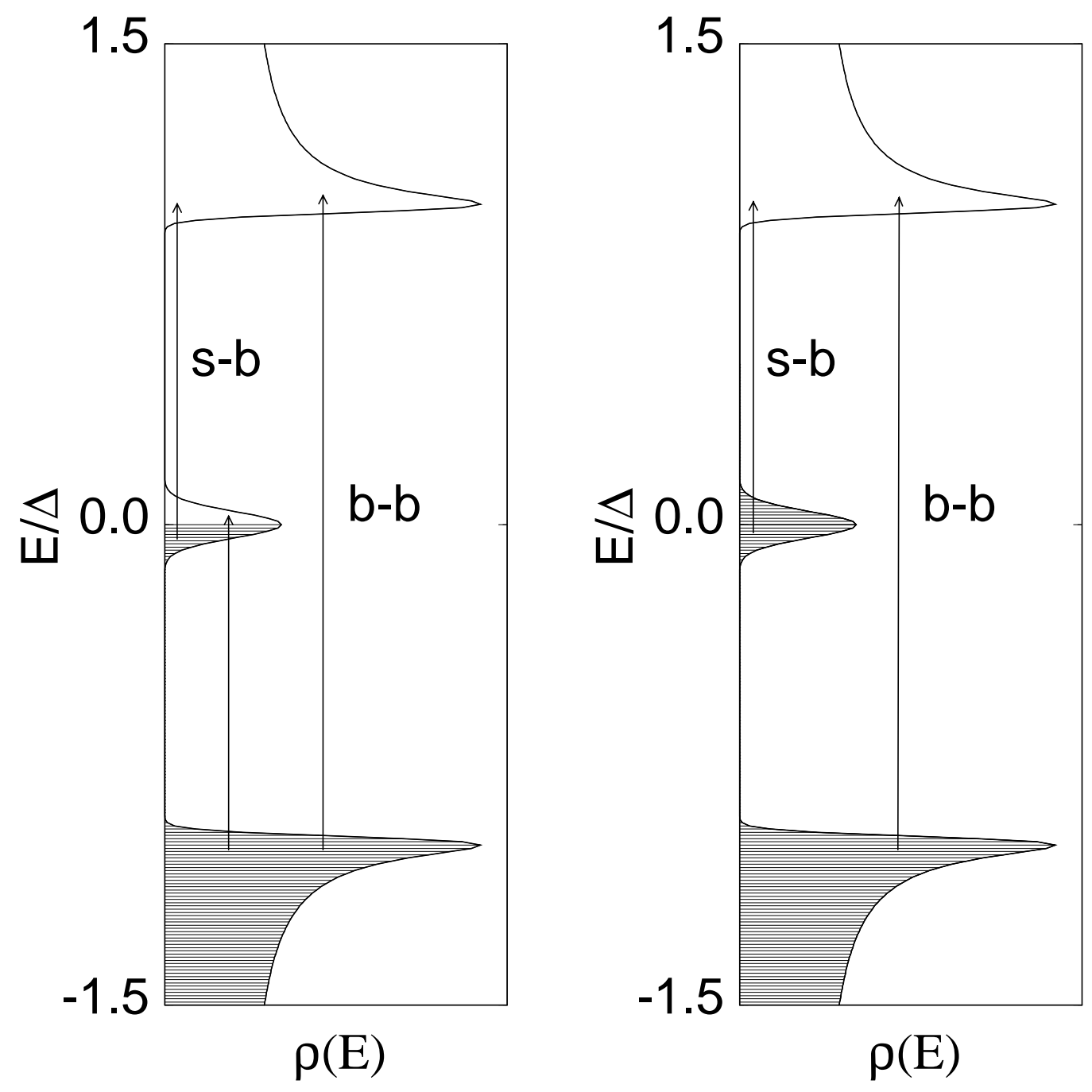

Fig. 3 


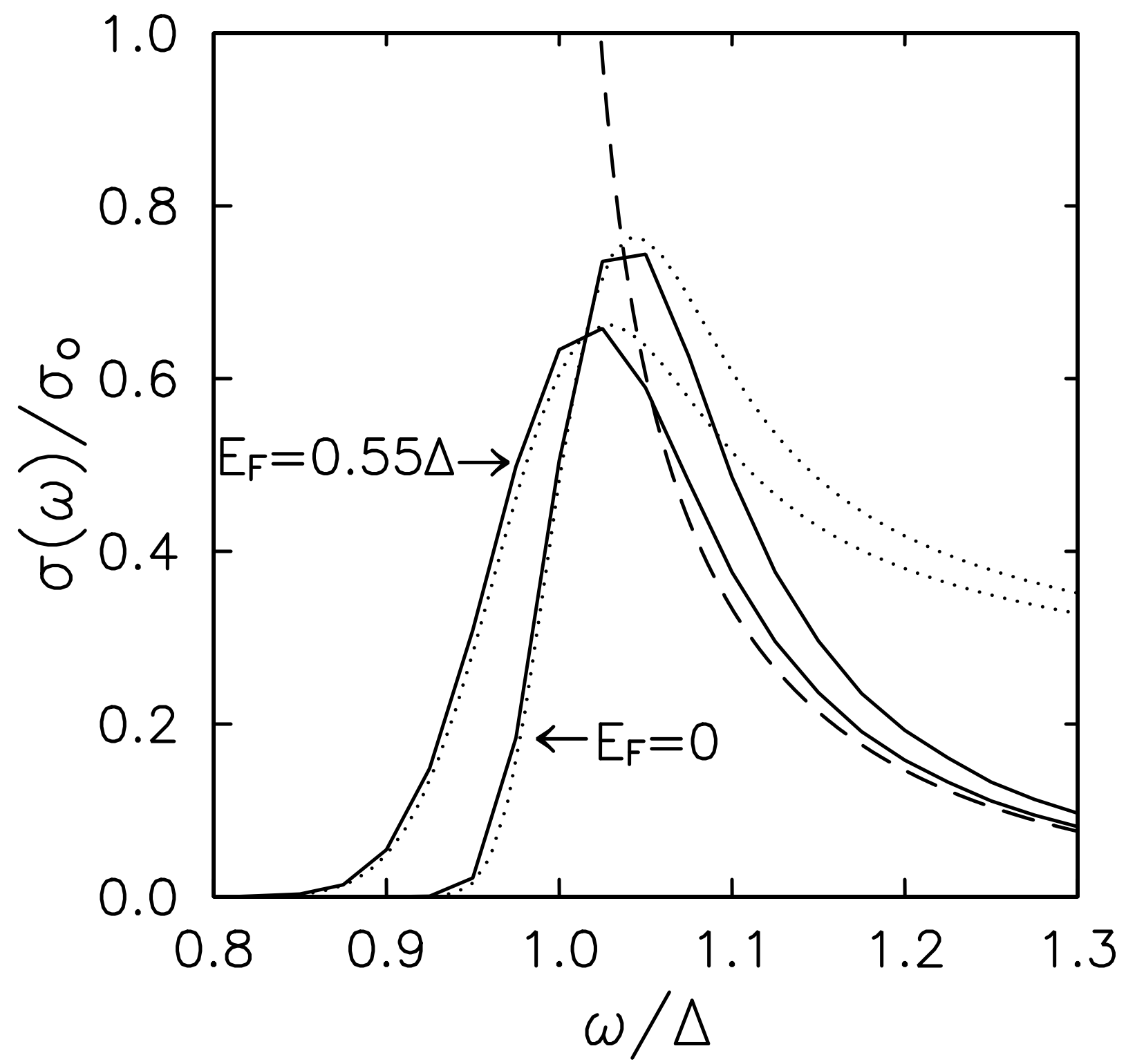

Fig. 4 

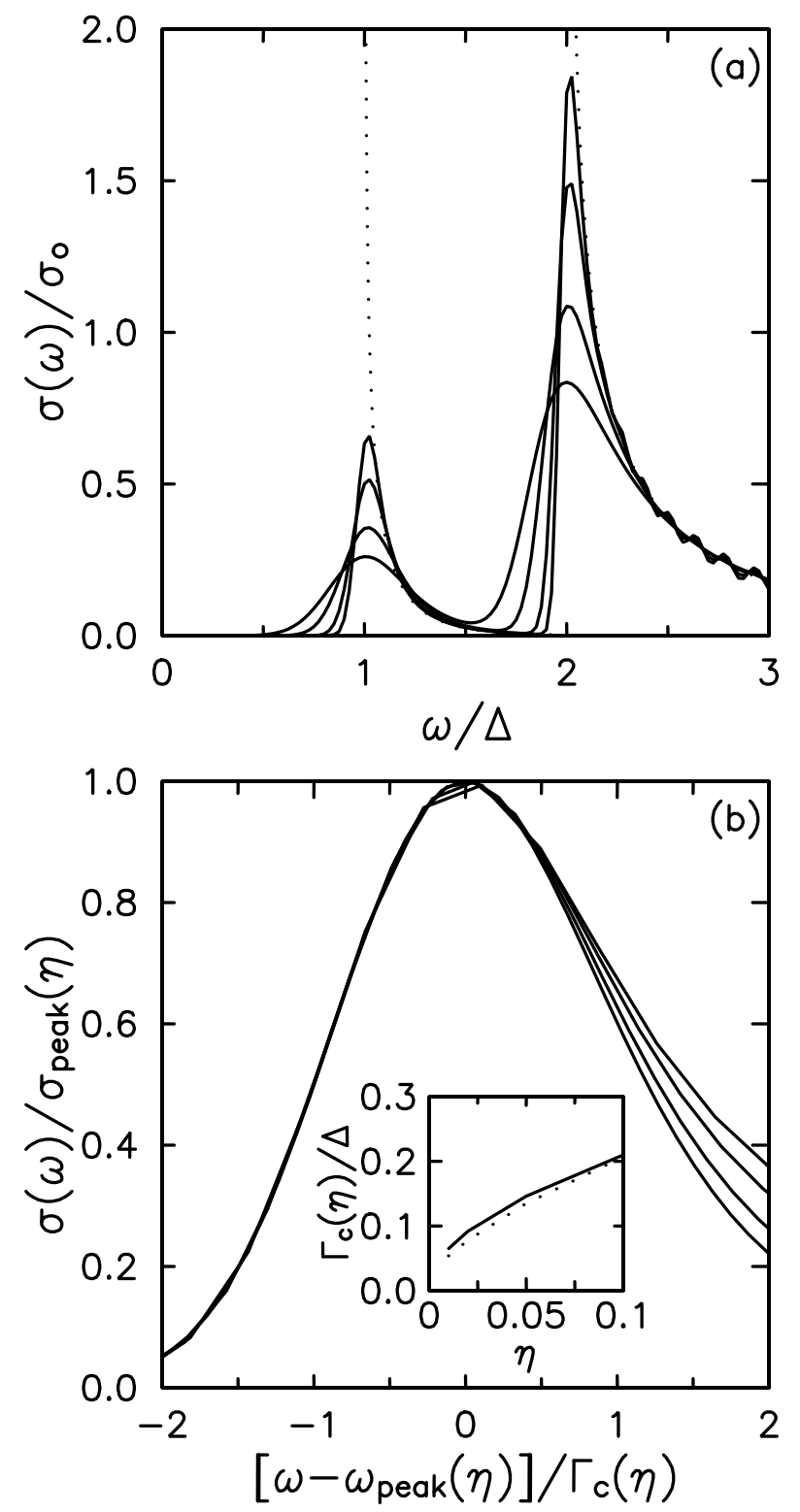

Fig. 5 